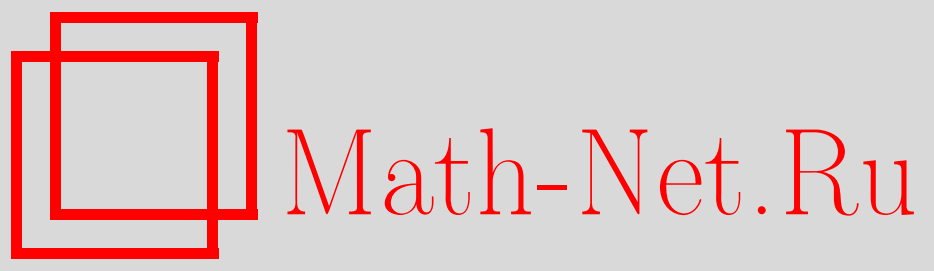

А. Д. Суханов, О. Н. Голубева, К квантовому обобщению равновесной статистической термодинамики. $(\hbar-k)$ Динамика, ТМФ, 2009, том 160, номер 2, 370-384

DOI: https://doi.org/10.4213/tmf6404

Использование Общероссийского математического портала Math-Net.Ru подразумевает, что вы прочитали и согласны с пользовательским соглашением http://www . mathnet.ru/rus/agreement

Параметры загрузки:

IP : 44.207 .124 .84

26 апреля 2023 г., 12:53:06

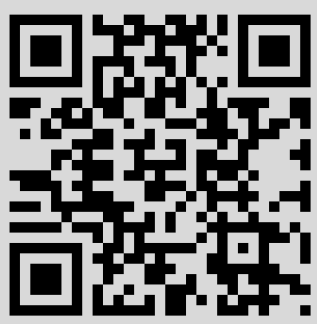




\title{
ФИЗИКА
}

Том 160, № 2

август, 2009

2009 г.

\author{
А. Д. Суханов ${ }^{* \dagger}$, О. Н. Голубева
}

\section{К КВАНТОВОМУ ОБОБЩЕНИЮ РАВНОВЕСНОЙ СТАТИСТИЧЕСКОЙ ТЕРМОДИНАМИКИ. $(\hbar-k)$-ДИНАМИКА}

\begin{abstract}
Показано, что квантовая статистическая механика (KCM) при описании квантовых и тепловых свойств объектов имеет смысл только своеобразного квазиклассического приближения. Предложено более общее, чем в КСМ, микроописание объектов в термостате с явным учетом вакуума, названное $(\hbar-k)$-динамикой. Сформулирована качественно новая модель окружения объекта QT-стат. Исследованы ее свойства, включая случаи "холодного" и "теплого" вакуумов. Введен оператор стохастического воздействия и показана его фундаментальная роль в микроописании. Установлено, что соответствующий макропараметр - эффективное воздействие - играет столь же существенную роль в макроописании. Через него выражаются важнейшие эффективные макропараметры равновесной квантовой статистической термодинамики - внутренняя энергия, температура и энтропия.
\end{abstract}

Ключевые слова: $(\hbar-k)$-динамика, QT-стат, "холодный” и “теплый вакуум", оператор стохастического воздействия, эффективное воздействие, квантово-тепловая энтропия.

\section{1. ВОЗМОЖНЫЕ ПОДХОДЫ К ПОСЛЕДОВАТЕЛЬНОМУ КВАНТОВО-ТЕПЛОВОМУ ОПИСАНИЮ ОБЪЕКТОВ ПРИРОДЫ}

Довольно долго господствовало убеждение, согласно которому равновесная квантовая статистическая механика (KCM) представляет собой не только адекватное описание микрообъектов в термостате, но и образует фундамент соответствующего макроописания. Иначе говоря, было принято считать, что КСМ позволяет вывести из микроописания термодинамические макропараметры и установить наблюдаемые взаимосвязи между ними (начала термодинамики, уравнения состояния и т.п.) [1]. Вместе с тем известно, что существуют такие макропараметры, например температура, аналоги которых на микроуровне до сих пор не рассматривались. Это привело к выдвижению идеи определенной самостоятельности и равноправия микро- и макроописаний природы при наличии некоторой взаимосвязи между ними [2], [3].

* Объединенный институт ядерных исследований, г. Дубна, Московская обл., Россия

${ }^{\dagger}$ Российский университет дружбы народов, Москва, Россия. E-mail: ogol@oldi.ru 
Проанализируем кратко проявившиеся к сегодняшнему дню элементы ограниченности равновесной КСМ как теории, претендующей на последовательное описание всех квантовых и тепловых явлений. В основе КСМ, как известно, лежит понятие матрицы (оператора) плотности, которая в энергетическом представлении имеет вид квантового канонического распределения Гиббса-фон Неймана

$$
w_{n}=e^{\left(F-\varepsilon_{n}\right) / \Theta},
$$

где $\varepsilon_{n}$ - спектр энергии объекта, $F$ - свободная энергия, определяемая условием нормировки, а $\Theta$ - обратный модуль распределения. Он имеет смысл множителя Лагранжа при выводе распределения (1) из принципа максимума энтропии в условиях теплового равновесия [4].

Ограниченность КСМ проявляется в трех отношениях. Во-первых, распределение (1) нечувствительно к учету вклада энергии нулевых колебаний $\varepsilon_{0}=\hbar \omega / 2$ в энергию объекта, поскольку такой учет автоматически компенсируется изменением выражения для свободной энергии $F$ вследствие условия нормировки. Между тем на опыте при сверхнизких температурах вклад энергии $\varepsilon_{0}$ - это вполне наблюдаемый эффект. Более того, как видно из модели квантового осциллятора, пренебрежение вкладом $\varepsilon_{0}$ равносильно пренебрежению принципиальной для квантовой физики величиной $\hbar / 2$ в правой части соотношения неопределенностей Гейзенберга. Действительно, в основном состоянии квантового осциллятора

$$
\Delta p_{0} \Delta q_{0}=\frac{\hbar}{2}=\frac{\varepsilon_{0}}{\omega}
$$

что подтверждает непосредственную связь между величинами $\varepsilon_{0}$ и $\hbar / 2$.

Во-вторых, в КСМ предполагается, что температура объекта не флуктуирует, тогда как для малых объектов, включая наночастицы, при сверхнизких температурах флуктуации температуры на опыте достаточно заметны [5]. Кроме того, сегодня вызывает серьезные сомнения автоматически следующее из распределения (1) утверждение о равенстве нулю минимального значения энтропии [6].

В-третьих, отметим еще одну внутреннюю непоследовательность КСМ. Согласно формуле (1) в качестве обратного модуля распределения для любых объектов при любых температурах в КСМ используется выражение

$$
\Theta=k_{\mathrm{B}} T .
$$

Это соответствует выбору классической модели термостата [7], [8] как совокупности слабо связанных классических осцилляторов. Однако в такой термостат помещается микрообъект с квантованной энергией, даже в условиях, когда $k_{\mathrm{B}} T<\left(\varepsilon_{n}-\varepsilon_{n-1}\right)$.

Иными словами, равновесная КСМ, основанная на распределении (1), не является последовательной теорией ни для квантовых, ни для тепловых явлений. Поэтому сегодня ее можно рассматривать лишь как своеобразное квазиклассическое приближение и по квантовым, и по тепловым характеристикам, определяемым соответственно постоянными Планка $\hbar$ и Больцмана $k_{\mathrm{B}}$.

Конечно, нельзя полностью исключить возможность того, что ограниченность стандартной КСМ будет преодолена путем усовершенствования аппарата, основанного на операторе плотности для смешанных состояний. Однако, на наш взгляд, 
для достижения последовательного квантово-теплового описания объектов природы возможны и два иных, отличных от КCM, подхода. В их основе, тем не менее, лежит одна общая идея - замена классической модели термостата на адекватную квантовую модель, или кванто-термостат (QТ-стат) [9]. В этой модели тепловое равновесие между объектом и окружением характеризуется эффективной температурой $T_{\text {eff }}[10]$, имеющей ненулевое ограничение снизу. Эта величина учитывает совместное стохастическое воздействие окружения квантового и теплового типов.

При первом из этих подходов можно модифицировать макроописание объектов в термостате путем учета квантовых эффектов в рамках статистической термодинамики Эйнштейна (см. [11] и гл. 9 в [12]) с учетом флуктуаций температуры, но без использования операторного формализма. В этом случае, основываясь на интуитивных соображениях и физических аналогиях, мы приходим к квантовой статистической термодинамике [10] как феноменологической теории для макроописания объектов природы в условиях равновесия с окружением (включая случай $T=0$ ).

Заметим, однако, что вполне допустим и второй подход, в рамках которого можно модифицировать фундаментальное микроописание тех же объектов в условиях теплового равновесия. Для этих целей предлагается сформулировать квантово-тепловую динамику, или кратко $(\hbar-k)$-динамику $(\hbar k \mathrm{D})$, как модификацию стандартной квантовой механики с учетом тепловых эффектов. Принципиальное отличие такой теории от KCM состоит в том, что в ней в условиях контакта с QT-статом состояние микрообъекта в общем случае описывается не матрицей плотности, а комплексной волновой функцией, зависящей от температуры.

В настоящей статье, являющейся продолжением работы [13], в которой были введены исходные понятия $\hbar k \mathrm{D}$, эта теория развивается далее как микроописание объекта в условиях теплового равновесия. В ее основу положен новый микропараметр - оператор стохастического воздействия. При этом продемонстрировано, что при усреднении соответствующих микропараметров по волновой функции, зависящей от температуры, можно получить важнейшие эффективные макропараметры, включая внутреннюю энергию, температуру и энтропию. Они будут иметь физический смысл стандартных термодинамических величин, характерных для феноменологического макроописания.

\section{2. МОДЕЛЬ QТ-СТАТА. “ХОЛОДНЫЙ” ВАКУУМ}

При построении $\hbar k \mathrm{D}$ мы исходим из того, что изолированных объектов в природе не существует. Иными словами, следуем модели Фейнмана [8], согласно которой любую систему можно представлять как совокупность исследуемого объекта и его окружения ("the rest of the Universe"). Окружение может оказывать на объект как регулярное, так и стохастическое воздействия. В данной работе мы исследуем только стохастическое воздействие. К нему можно отнести два типа воздействия квантовое и тепловое, характеризуемые постоянными Планка и Больцмана соответственно.

Для описания окружения, оказывающего целостное стохастическое воздействие и названного ранее Умэдзавой [14] термополевым вакуумом, мы вводим конкретную модель - QТ-стат. Она является естественным обобщением модели классического 
термостата, используемого в стандартных теориях тепловых явлений [1]. В соответствии с этим QT-стат - это совокупность слабо связанных квантовых осцилляторов всех возможных частот. Прообразом такой модели в природе может служить равновесное тепловое излучение.

Особенность нашего понимания этой модели состоит в том, что мы считаем необходимым применять ее как к "теплому" $(T \neq 0)$, так и к “холодному" $(T=0)$ вакуумам. Тем самым, в духе Эйнштейна (см. [15]) мы исходим из более общего понимания теплового равновесия, которое в принципе может установиться при любом типе стохастического воздействия окружения (чисто квантовом, квантово-тепловом и чисто тепловом).

Начнем изложение с рассмотрения "холодного" вакуума и обсудим с новой точки зрения описание одиночного квантового осциллятора из числа осцилляторов, образующих модель QТ-стата при $T=0$. В целях будущего обобщения на случай $T \neq 0$ наиболее пригодными оказываются не его известные собственные состояния $\Psi_{n}(q)$ в $q$-представлении, а когерентные состояния (KC) [16].

Напомним, однако, что низшее состояние в обоих наборах состояний одинаково. В представлении чисел заполнения этому состоянию соответствует "холодный" вакуум, в котором число частиц $n=0$. В свою очередь, в $q$-представлении то же самое основное состояние квантового осциллятора описывается вещественной волновой функцией

$$
\Psi_{0}(q)=\left[2 \pi\left(\Delta q_{0}\right)^{2}\right]^{-1 / 4} e^{-q^{2} / 4\left(\Delta q_{0}\right)^{2}} .
$$

Вследствие свойств распределения Гаусса фурье-образ этой функции $\Psi_{0}(p)$ имеет аналогичный вид (с заменой $q$ на $p$ ), причем дисперсии координаты и импульса равны соответственно

$$
\left(\Delta p_{0}\right)^{2}=\frac{\hbar m \omega}{2}, \quad\left(\Delta q_{0}\right)^{2}=\frac{\hbar}{2 m \omega} .
$$

Как известно, КС являются собственными состояниями неэрмитова оператора уничтожения частиц $\hat{a}$ с комплексными собственными значениями. Однако среди них есть одно выделенное состояние $\left|0_{a}\right\rangle$ вакуума частиц, в котором собственное значение оператора $\hat{a}$ равно нулю:

$$
\hat{a}\left|0_{a}\right\rangle=0\left|0_{a}\right\rangle
$$

В дальнейшем нам будет удобнее описывать QТ-стат в $q$-представлении. Поэтому выразим операторы уничтожения $\hat{a}$ и рождения $\hat{a}^{\dagger}$ традиционным способом через операторы $\hat{p}$ и $\hat{q}$. Имеем

$$
\hat{a}=\frac{1}{2}\left(\frac{\hat{p}}{\sqrt{\Delta p_{0}^{2}}}-i \frac{\hat{q}}{\sqrt{\Delta q_{0}^{2}}}\right), \quad \hat{a}^{\dagger}=\frac{1}{2}\left(\frac{\hat{p}}{\sqrt{\Delta p_{0}^{2}}}+i \frac{\hat{q}}{\sqrt{\Delta q_{0}^{2}}}\right) .
$$

Тогда оператор числа частиц принимает вид

$$
\hat{N}_{a}=\hat{a}^{\dagger} \hat{a}=\frac{1}{\hbar \omega}\left(\frac{\hat{p}^{2}}{2 m}+\frac{m \omega^{2} \hat{q}^{2}}{2}-\frac{\hbar \omega}{2} \hat{I}\right),
$$

где $\hat{I}$ - единичный оператор. 
Сумма первого и второго слагаемых в последней скобке образует гамильтониан $\mathcal{H}$ квантового осциллятора, так что после домножения соотношения (8) слева и справа на $\hbar \omega$ получим стандартную взаимосвязь выражений для гамильтониана в $q$ и $n$-представлениях:

$$
\hat{\mathcal{H}}=\frac{\hat{p}^{2}}{2 m}+\frac{m \omega^{2} \hat{q}^{2}}{2}=\hbar \omega\left(\hat{N}_{a}+\frac{1}{2} \hat{I}\right) .
$$

$\mathrm{C}$ точки зрения термодинамики нас интересует внутренняя энергия квантового осциллятора, находящегося в равновесии с “холодным" QT-статом. Ее величина равна среднему от гамильтониана по состоянию $\left|0_{a}\right\rangle \equiv\left|\Psi_{0}(q)\right\rangle$ :

$$
U_{0}=\left\langle\Psi_{0}(q)|\hat{\mathcal{H}}| \Psi_{0}(q)\right\rangle=\hbar \omega\left\langle\Psi_{0}(q)\left|\hat{N}_{a}\right| \Psi_{0}(q)\right\rangle+\frac{\hbar \omega}{2}=\frac{\hbar \omega}{2}=\varepsilon_{0}
$$

Из формулы (10) следует, что в данном случае состояние без частиц совпадает с состоянием гамильтониана с минимальной энергией $\varepsilon_{0}$. Сама же величина $\varepsilon_{0}$, традиционно трактуемая как энергия нулевых колебаний, приобретает физический смысл внутренней энергии $U_{0}$ квантового осциллятора в равновесии с "холодным" вакуумом.

\section{3. МОДЕЛЬ QТ-СТАТА. ПЕРЕХОД К “ТЕПЛОМУ” ВАКУУМУ}

Переход от "холодного" к "теплому" вакууму в духе Умэдзавы можно осуществить с помощью $(u, v)$-преобразования Боголюбова с коэффициентами, зависящими от температуры:

$$
u=\left(\frac{1}{2} \operatorname{cth} \frac{\hbar \omega}{2 k_{\mathrm{B}} T}+\frac{1}{2}\right)^{1 / 2} e^{i \pi / 4}, \quad v=\left(\frac{1}{2} \operatorname{cth} \frac{\hbar \omega}{2 k_{\mathrm{B}} T}-\frac{1}{2}\right)^{1 / 2} e^{-i \pi / 4} .
$$

В данном случае указанное преобразование является каноническим, но приводит к унитарно-неэквивалентному представлению, поскольку QТ-стат при любой температуре - это система с бесконечно большим числом степеней свободы.

В конечном итоге такое преобразование сводится к переходу от совокупности КС квантового осциллятора к более общей совокупности состояний, называемых тепловыми коррелированно-когерентными состояниями (ТKKC) [14]. Они выделены тем, что обеспечивают насыщение соотношения неопределенностей Шредингера "координата-импульс" при любой температуре. С точки зрения аппарата вторичного квантования $(u, v)$-преобразование Боголюбова обеспечивает переход от исходной системы частиц с "холодным" вакуумом $\left|0_{a}\right\rangle$ к системе квазичастиц, описываемой операторами уничтожения $\hat{b}$ и рождения $\hat{b}^{\dagger}$ с “теплым" вакуумом $\left|0_{b}\right\rangle$. При этом выбор (11) коэффициентов преобразования фиксируется требованием, чтобы при любом способе описания выражение для средней энергии квантового осциллятора в тепловом равновесии определялось формулой Планка, которую можно получить из эксперимента:

$$
\mathcal{E}_{\mathrm{Pl}}=\hbar \omega\left(e^{\hbar \omega / k_{\mathrm{B}} T}-1\right)^{-1}+\frac{\hbar \omega}{2}=\frac{\hbar \omega}{2} \operatorname{cth} \frac{\hbar \omega}{2 k_{\mathrm{B}} T}
$$


Как было показано в работе [13], состоянию "теплого" вакуума $\left|0_{b}\right\rangle \equiv\left|\Psi_{T}(q)\right\rangle$ в $q$-представлении соответствует комплексная волновая функция

$$
\Psi_{T}(q)=\left[2 \pi(\Delta q)^{2}\right]^{-1 / 4} \exp \left\{-\frac{q^{2}}{4(\Delta q)^{2}}(1-i \alpha)\right\},
$$

где

$$
(\Delta q)^{2}=\frac{\hbar}{2 m \omega} \operatorname{cth} \frac{\hbar \omega}{2 k_{\mathrm{B}} T}, \quad \alpha=\left[\operatorname{sh} \frac{\hbar \omega}{2 k_{\mathrm{B}} T}\right]^{-1} .
$$

Для ее фурье-образа $\Psi_{T}(p)$ справедливо аналогичное выражение с тем же коэффициентом $\alpha$ и

$$
(\Delta p)^{2}=\frac{\hbar m \omega}{2} \operatorname{cth} \frac{\hbar \omega}{2 k_{\mathrm{B}} T} .
$$

Заметим, что выражения для плотностей вероятности $\rho_{T}(q)$ и $\rho_{T}(p)$ были получены еще Блохом [17]. Однако выражения для фаз, зависящих от параметра $\alpha$, которые играют весьма существенную роль, ранее известны не были. Нетрудно также видеть, что при $T \rightarrow 0$ параметр $\alpha \rightarrow 0$, а сама функция $\Psi_{T}(q)$ из совокупности TKKC переходит в функцию $\Psi_{0}(q)$ из совокупности KC.

Разумеется, состояния из ТKКС являются собственными состояниями неэрмитова оператора уничтожения квазичастиц $\hat{b}$ с комплексными собственными значениями. Среди них также есть одно выделенное состояние вакуума квазичастиц, в котором собственное значение оператора $\hat{b}$ равно нулю,

$$
\hat{b}\left|0_{b}\right\rangle=0\left|0_{b}\right\rangle .
$$

Пользуясь условием (16) и выражением (13) для волновой функции "теплого" вакуума, находим выражение для оператора $\hat{b}$ в $q$-представлении:

$$
\hat{b}=\frac{1}{2}\left[\frac{\hat{p}}{\sqrt{\Delta p_{0}^{2}}}-i \frac{\hat{q}}{\sqrt{\Delta q_{0}^{2}}}\left(\operatorname{cth} \frac{\hbar \omega}{2 k_{\mathrm{B}} T}\right)^{-1}(1-i \alpha)\right] .
$$

Соответствующий оператор рождения квазичастиц имеет вид

$$
\hat{b}^{\dagger}=\frac{1}{2}\left[\frac{\hat{p}}{\sqrt{\Delta p_{0}^{2}}}+i \frac{\hat{q}}{\sqrt{\Delta q_{0}^{2}}}\left(\operatorname{cth} \frac{\hbar \omega}{2 k_{\mathrm{B}} T}\right)^{-1}(1+i \alpha)\right] .
$$

Можно убедиться, что при $T \rightarrow 0$ операторы $\hat{b}^{\dagger}$ и $\hat{b}$ для квазичастиц переходят в операторы $\hat{a}^{\dagger}$ и $\hat{a}$ для частиц.

Действуя, как и выше, получим выражение для оператора числа квазичастиц в $q$-представлении

$$
\hat{N}_{b}=\hat{b}^{\dagger} \hat{b}=\frac{1}{4}\left[\frac{\hat{p}^{2}}{\Delta p_{0}^{2}}+\frac{\hat{q}^{2}}{\Delta q_{0}^{2}}-2\left(\operatorname{cth} \frac{\hbar \omega}{2 k_{\mathrm{B}} T}\right)^{-1}\left(\hat{I}+\frac{\alpha}{\hbar}\{\hat{p}, \hat{q}\}\right)\right],
$$

где при вычислении второго слагаемого было учтено, что $1+\alpha^{2}=\operatorname{cth}\left(\hbar \omega / 2 k_{\mathrm{B}} T\right)$. 
Переходя от оператора числа квазичастиц к исходному гамильтониану, после домножения на $\hbar \omega$ получим

$$
\hat{\mathcal{H}}=\hbar \omega\left[\hat{N}_{b}+\frac{1}{2}\left(\operatorname{cth} \frac{\hbar \omega}{2 k_{\mathrm{B}} T}\right)^{-1}\left(\hat{I}+\frac{\alpha}{\hbar}\{\hat{p}, \hat{q}\}\right)\right] .
$$

Подчеркнем, что входящий в формулу (20) оператор $\{\hat{p}, \hat{q}\}$ также можно выразить через билинейные комбинации операторов $\hat{b}^{\dagger}$ и $\hat{b}$, но они будут отличаться от оператора числа квазичастиц. Это означает, что операторы $\hat{\mathcal{H}}$ и $\hat{N}_{b}$ не коммутируют, так что волновая функция вида (13), характеризующая состояние “теплого" вакуума, не является собственной функцией гамильтониана.

Нас по-прежнему интересует термодинамическая величина - внутренняя энергия $U$ квантового осциллятора, находящегося теперь в тепловом равновесии с "теплым" QТ-статом. Вычисляя ее, как и в разделе 2, в $q$-представлении получим

$$
\begin{aligned}
U=\left\langle\Psi_{T}(q)|\hat{\mathcal{H}}| \Psi_{T}(q)\right\rangle= & \hbar \omega\left[\left\langle\Psi_{T}(q)\left|\hat{N}_{b}\right| \Psi_{T}(q)\right\rangle\right]+ \\
& +\frac{\hbar \omega}{2 \operatorname{cth}\left(\hbar \omega / 2 k_{\mathrm{B}} T\right)}\left(1+\frac{\alpha}{\hbar}\left\langle\Psi_{T}(q)|\{p, q\}| \Psi_{T}(q)\right\rangle\right) .
\end{aligned}
$$

Поскольку в формуле (21) усреднение ведется по вакууму квазичастиц, первое слагаемое в ней обращается в нуль. В то же время в работе [13] было показано, что

$$
\left\langle\Psi_{T}(q)|\{\hat{p}, \hat{q}\}| \Psi_{T}(q)\right\rangle=\hbar \alpha .
$$

В результате для внутренней энергии квантового осциллятора в “теплом" QТ-стате в рамках $\hbar k \mathrm{D}$ получаем

$$
U=\frac{\hbar \omega}{2 \operatorname{cth}\left(\hbar \omega / 2 k_{\mathrm{B}} T\right)}\left(1+\alpha^{2}\right)=\frac{\hbar \omega}{2} \operatorname{cth} \frac{\hbar \omega}{2 k_{\mathrm{B}} T}=\mathcal{E}_{\mathrm{Pl}},
$$

где $\mathcal{E}_{\mathrm{Pl}}$ определяется формулой Планка (12). Это означает, что средняя энергия квантового осциллятора при $T \neq 0$ имеет термодинамический смысл его внутренней энергии в случае равновесия с "теплым" QT-статом. При $T \rightarrow 0$ она переходит в аналогичную величину, соответствующую равновесию с "холодным" QT-статом.

Хотя окончательный результат (23) получился вполне ожидаемым, из него следует ряд существенных выводов.

1. В отличие от вычисления внутренней энергии в $\mathrm{KCM}$, где все определяется плотностью вероятности $\rho_{T}(q)$, в $\hbar k \mathrm{D}$ в то же самое выражение дает существенный вклад квадрат параметра $\alpha$, определяющего фазу волновой функции, что свидетельствует о более последовательном использовании в этой теории квантовой идеологии.

2. В $\hbar k \mathrm{D}$ выражение для $\operatorname{cth}\left(\hbar \omega / 2 k_{\mathrm{B}} T\right)$, входящее в формулу $(23)$, появляется в ней как целостная величина, тогда как в KCM вклад $\varepsilon_{0}$ в ту же формулу обычно возникает отдельно как добавочная, не имеющая термодинамического смысла величина, и потому часто вообще не учитывается.

3 . В $\hbar k \mathrm{D}$ операторы $\hat{\mathcal{H}}$ и $\hat{N}_{b}$ не коммутируют, поскольку гамильтониан содержит комбинации операторов типа $\hat{b}^{\dagger} \hat{b}^{\dagger}$ и $\hat{b} \hat{b}$. Их присутствие свидетельствует о несохранении числа квазичастиц, что характерно для ситуации со спонтанным нарушением 
симметрии [18]. По нашему мнению, предложенная модель QТ-стата - это универсальная модель окружения, оказывающего стохастическое воздействие на объект. Поэтому проявления спонтанного нарушения симметрии в природе вовсе не должны ограничиваться феноменами сверхтекучести и сверхпроводимости.

\section{4. ОПЕРАТОР СТОХАСТИЧЕСКОГО ВОЗДЕЙСТВИЯ}

Эффективное воздействие как макропараметр было постулировано ранее в рамках квантовой статистической термодинамики [10] путем обобщения представлений об адиабатических инвариантах [19]. Развиваемое здесь последовательное микроописание объекта в тепловом равновесии в рамках $\hbar k \mathrm{D}$ будем основывать на модели QT-стата, описываемой волновой функцией вида (13).

Поскольку исходным положением $\hbar k \mathrm{D}$ является идея целостного стохастического воздействия на объект со стороны QT-стата, для ее реализации введем новый оператор в гильбертовом пространстве состояний микрообъекта. В качестве наводящих соображений воспользуемся анализом правой части соотношения неопределенностей Шредингера "координата-импульс"

$$
(\Delta p)^{2}(\Delta q)^{2}=\left|\widetilde{R}_{p q}\right|^{2}
$$

Для любого объекта, а не только квантового осциллятора в термостате, комплексная величина в правой части (24)

$$
\widetilde{R}_{p q}=\langle\Delta p \mid \Delta q\rangle=\langle|\Delta \hat{p} \Delta \hat{q}|\rangle
$$

имеет двоякий смысл. С одной стороны, она представляет собой амплитуду перехода из состояния $|\Delta q\rangle$ в состояние $|\Delta p\rangle$, с другой стороны, ее можно трактовать как среднее или квантовый коррелятор Шредингера по произвольному состоянию $\mid>$ от некоторого оператора.

Как известно, отличие от нуля величины (25) является основополагающим признаком неклассической теории, в которой существенную роль играет стохастическое воздействие окружения на объект. Поэтому вполне естественно допустить, что стоящий под знаком среднего в формуле (25) оператор сам имеет фундаментальный смысл. С учетом соображений размерности назовем его оператором стохастического воздействия,

$$
\hat{j} \equiv \Delta \hat{p} \Delta \hat{q}
$$

Разумеется, следует помнить, что операторы $\Delta \hat{q}$ и $\Delta \hat{p}$ между собой не коммутируют, а их произведение представляет собой неэрмитов оператор.

Для дальнейшего анализа, следуя Шредингеру (см. с. 473 в [20]), представим данный оператор в виде

$$
\hat{j}=\frac{1}{2}\langle|\Delta \hat{p} \Delta \hat{q}+\Delta \hat{q} \Delta \hat{p}|\rangle+\frac{1}{2}\langle|\Delta \hat{p} \Delta \hat{q}-\Delta \hat{q} \Delta \hat{p}|\rangle=\hat{\sigma}-i \hat{j}_{0},
$$


что позволяет отделить в нем эрмитову часть (оператор $\hat{\sigma}$ ) от антиэрмитовой, в которой эрмитов оператор

$$
\hat{j}_{0} \equiv \frac{i}{2}[\hat{p}, \hat{q}]=\frac{\hbar}{2} \hat{I}
$$

Нетрудно видеть, что среднее значение $\sigma=\langle|\hat{\sigma}|\rangle$ оператора $\hat{\sigma}$ напоминает выражение для стандартного коррелятора флуктуаций координаты и импульса из классической теории вероятностей, в которое оно переходит при замене операторов $\Delta \hat{q}$ и $\Delta \hat{p}$ на $c$-числа. Оно отражает вклад в амплитуду перехода $\widetilde{R}_{p q}$ стохастического воздействия окружения. Поэтому впредь будем называть оператор $\hat{\sigma}$ оператором внешнего воздействия. Ранее возможность использовать подобный оператор обсуждалась в работе Боголюбова и Крылова [7]. В ней он рассматривался как квантовый аналог классической переменной действия в совокупности переменных действие-угол.

В то же время как оператор $\hat{j}_{0}$, так и оператор $\hat{j}$ в целом ранее не вводились. Оператор вида (28) отражает специфическую особенность объектов независимо от их состояния "чувствовать" минимальное стохастическое воздействие "холодного" вакуума и адекватно реагировать на него. Поэтому его следует трактовать как оператор минимального стохастического воздействия. Его среднее значение $J_{0}=$ $\hbar / 2$ не зависит от выбора состояния, по которому производится усреднение, так что оно имеет смысл инвариантного собственного значения оператора $\hat{j}_{0}$.

Отсюда следует, что в данном случае мы имеем дело с универсальной величиной $J_{0}$, которую мы будем называть минимальным воздействием. Ее фундаментальный характер определяется уже тем, что она связана с мировой постоянной Планка $\hbar$. Однако этим все не исчерпывается. Действительно, согласно традиции, восходящей к Планку, саму величину $\hbar$ принято называть элементарным квантом действия. В то же время в величине $J_{0}$ существенную роль играет множитель $1 / 2$, тогда как половины кванта действия в природе не наблюдается. Тем самым, по нашему мнению, величины $\hbar$ и $\hbar / 2$, совпадая по размерности, имеют различный физический смысл и потому должны именоваться по-разному. С этой точки зрения было бы более естественно называть величину $\hbar$ квантом внешнего воздействия.

Таким образом, величина $\hbar$ - это минимальная порция воздействия, передаваемого объекту от внешнего окружения или от другого объекта. Поэтому фотон и другие кванты полей - переносчиков фундаментальных взаимодействий, прежде всего, являются переносчиками минимального воздействия, равного ћ. То же самое относится, конечно, и к фононам.

Наконец, заметим, что только величина $\hbar$ связана с дискретностью спектра энергии квантового осциллятора в отсутствие термостата. В то же время величина $\hbar / 2$ имеет самостоятельный физический смысл, определяя согласно формуле (10) минимальное значение макропараметра - внутренней энергии $U_{0}$ квантового осциллятора в "холодном" QТ-стате (при $T=0)$.

Оценим особенности оператора стохастического воздействия, используемого ниже в микроописании. Напомним, что этот оператор является неэрмитовым. Это обстоятельство, казалось бы, противоречит стандартным требованиям, предъявляемым к операторам наблюдаемых в квантовой механике. Однако на самом деле 
в этом факте нет ничего необычного. Конечно, для эрмитовых операторов характерно наличие собственных состояний с вещественными собственными значениями, которые принято сопоставлять наблюдаемым величинам. Но с физической точки зрения такие собственные значения не столь уж интересны, ибо они характеризуют нечто неизменное, как, например, стационарные состояния.

Другое дело, если нас интересует подлинная квантовая динамика, которую естественно ассоциировать с переходами из одного состояния в другое. В этом случае важнейшую роль играют как раз неэрмитовы операторы. $\mathrm{K}$ числу наиболее известных из них относятся операторы рождения и уничтожения или, например, матрица рассеяния. В этом же ряду занимает свое место и оператор стохастического воздействия.

Чтобы продемонстрировать близость этого оператора к другим операторам квантовой механики, сравним его, например, со стандартным оператором импульса. Нетрудно убедиться в том, что собственное значение оператора импульса вещественно и отлично от нуля только в единственном случае, когда волновая функция представляет собой бесконечную гармоническую волну де Бройля. Однако, строго говоря, такая волновая функция вообе не принадлежит гильбертову пространству (напомним, что в импульсном представлении ее прообразом служит функция $\left.\delta\left(p-p_{0}\right)\right)$. Поэтому в общем случае оператор импульса, действуя на произвольную функцию $\Psi_{T}(q)$, просто переводит ее в другую функцию: $\Phi_{T}(q) \equiv \hat{p} \Psi_{T}(q)$. Тем самым оператор $\hat{p}$ фактически проявляет себя как оператор перехода из одного состояния в другое и не более того. Хотя оператор импульса и эрмитов, эта его особенность сказывается только в одном состоянии.

Таким образом, оператор $\hat{j}$ выделен только тем, что имеет смысл оператора перехода из одного состояния в другое в результате стохастического воздействия окружения на объект.

\section{5. ЭФФЕКТИВНОЕ ВОЗДЕЙСТВИЕ КАК ФУНДАМЕНТАЛЬНЫЙ МАКРОПАРАМЕТР. ВЗАИМОСВЯЗЬ С ВНУТРЕННЕЙ ЭНЕРГИЕЙ И ЭФФЕКТИВНОЙ ТЕМПЕРАТУРОЙ}

Перейдем к макроописанию объектов на основе их микроописания в рамках $\hbar k \mathrm{D}$. Нетрудно видеть, что среднее значение $\tilde{J}$ оператора $\hat{j}$ вида (27) совпадает с комплексной амплитудой перехода $\widetilde{R}_{p q}$ и в тепловом равновесии может быть представлено в виде

$$
\widetilde{J}=\left\langle\Psi_{T}(q)|\hat{j}| \Psi_{T}(q)\right\rangle=\sigma-i J_{0},
$$

где $\sigma$ и $J_{0}$ - средние значения соответствующих операторов. Модуль комплексной величины $\widetilde{J}$, равный

$$
|\tilde{J}|=\sqrt{\sigma^{2}+J_{0}^{2}}=\sqrt{\sigma^{2}+\frac{\hbar^{2}}{4}} \equiv J_{\mathrm{eff}},
$$

мы будем ниже рассматривать как новый макропараметр и называть его эффективным воздействием. Для квантового осциллятора он имеет вид

$$
J_{\mathrm{eff}}=\frac{\hbar}{2} \operatorname{cth} \frac{\hbar \omega}{2 k_{\mathrm{B}} T} .
$$


Эта величина совпадает с аналогичной величиной, постулированной ранее в работе [10] из интуитивных соображений.

Установим теперь взаимосвязь эффективного воздействия с традиционными термодинамическими величинами. Сравнивая выражение (31) для $J_{\text {eff }}$ и $(23)$ для внутренней энергии $U$ нетрудно видеть, что для квантового осциллятора

$$
U=\omega|\tilde{J}|=\omega J_{\mathrm{eff}}
$$

В пределе высоких температур, когда

$$
\sigma \rightarrow J_{T}=\frac{k_{\mathrm{B}} T}{\omega} \gg \frac{\hbar}{2}
$$

соотношение (32) принимает вид

$$
U=\omega J_{T}
$$

Эта формула была получена ранее Больцманом [19] для макропараметров в классической термодинамике путем обобщения представления об адиабатических инвариантах, используемых в классической механике.

Соотношение (32) позволяет также представить в явном виде взаимосвязь эффективного воздействия с эффективной температурой $T_{\text {eff }}[10]:$

$$
T_{\mathrm{eff}}=\frac{\omega}{k_{\mathrm{B}}} J_{\mathrm{eff}}
$$

Отсюда следует, что

$$
T_{\mathrm{eff}}^{\min }=\frac{\omega}{k_{\mathrm{B}}} J_{0}=\frac{\hbar \omega}{2 k_{\mathrm{B}}} \neq 0 .
$$

Наконец, заметим, что с помощью формул $(32),(14)$ и (15) соотношению неопределенностей Шредингера для квантового осциллятора при $T \neq 0$ можно придать вид, аналогичный выражению (2) для случая $T=0$,

$$
\Delta p \cdot \Delta q=|\tilde{J}|=\frac{U}{\omega} .
$$

Чтобы подчеркнуть роль макропараметра $J_{\text {eff }}$, дадим формуле (30) геометрическую трактовку. Напомним, что переход от основного состояния квантового осциллятора, принадлежащего КС, к состоянию “теплого" QТ-стата, принадлежащего TKKC, осуществляется с помощью $(u, v)$-преобразования Боголюбова. Оно образует группу Ли в пространстве состояний, локально изоморфную группе Лоренца в двумерном мире событий. Это означает, что в ТККС мы можем рассматривать совокупность $\left(J_{\mathrm{eff}}, \sigma\right)$ как двумерный времениподобный вектор в псевдоевклидовом пространстве состояний, а величину $J_{0}=\hbar / 2$ - как длину этого вектора или инвариант соответствующей группы [21]:

$$
J_{\text {eff }}^{2}-\sigma^{2}=J_{0}^{2}=\frac{\hbar^{2}}{4}=\text { inv } .
$$

Роль традиционных лоренцевых множителей $\beta$ и $\gamma$ в данном случае играют величины

$$
\beta_{T}=\left[\operatorname{ch} \frac{\hbar \omega}{2 k_{\mathrm{B}} T}\right]^{-1}, \quad \gamma_{T}=\operatorname{cth} \frac{\hbar \omega}{2 k_{\mathrm{B}} T} .
$$


Нетрудно видеть, что вектор эффективного воздействия $\left(J_{\text {eff }}, \sigma\right)$ представляет собой аналог двумерного вектора энергии-импульса $(\varepsilon, p c)$ в релятивистской механике:

$$
\varepsilon^{2}-p^{2} c^{2}=\varepsilon_{0}^{2}=m^{2} c^{4} .
$$

В частности, для квантового осциллятора в ТККС величина $J_{\text {eff }}$ является аналогом $\mathcal{E}$, а $\sigma$ - аналогом $p c$. Если учесть взаимосвязь (32) внутренней энергии квантового осциллятора с эффективным воздействием, то выражение (38) примет вид

$$
U^{2}-\sigma^{2} \omega^{2}=U_{0}^{2}=\left(\frac{\hbar \omega}{2}\right)^{2}
$$

По физическому смыслу формулы (41) и (38) полностью аналогичны соотношению (40), характеризующему так называемую массовую оболочку в двумерном пространстве событий.

\section{6. ЭФФЕКТИВНАЯ ЭНТРОПИЯ В $(\hbar-k)$-ДИНАМИКЕ}

Возможность введения энтропии в рамках $\hbar k \mathrm{D}$ также опирается на использование волновой функции вместо оператора плотности. Для определения этой величины в качестве исходного мы принимаем формальное выражение

$$
-k_{\mathrm{B}}\left\{\int \rho(q) \ln \rho(q) d q+\int \rho(p) \ln \rho(p) d p\right\},
$$

предложенное ранее в работе [22] (см. также [23]). Здесь $\rho(q)=|\Psi(q)|^{2}$ и $\rho(p)=$ $|\Psi(p)|^{2}$ - размерные вероятности в координатном и импульсном представлениях соответственно.

Используя выражение (13) для волновой функции квантового осциллятора, приведем $\rho(q)$ к безразмерному виду:

$$
\tilde{\rho}(\tilde{q})=\left[\operatorname{cth} \frac{\hbar \omega}{2 k_{\mathrm{B}} T}\right]^{-1} e^{-\tilde{q}^{2} / 2}, \quad \tilde{q}^{2}=\frac{q^{2}}{(\Delta q)^{2} \delta},
$$

где $\delta$ - произвольная константа. Аналогичное выражение для ее фурье-образа $\tilde{\rho}(\tilde{p})$ отличается лишь заменой $q$ на $p$.

Используя безразмерные выражения, мы предлагаем определить энтропию в рамках $\hbar k D$ равенством

$$
S_{q p}=-k_{\mathrm{B}}\left\{\int \tilde{\rho}(\tilde{q}) \ln \tilde{\rho}(\tilde{q}) d \tilde{q}+\int \tilde{\rho}(\tilde{p}) \ln \tilde{\rho}(\tilde{p}) d \tilde{p}\right\} .
$$

После подстановки в (44) соответствующих выражений для $\tilde{\rho}(\tilde{q})$ и $\tilde{\rho}(\tilde{p})$ получим

$$
S_{q p}=k_{\mathrm{B}}\left\{\left(1+\ln \frac{2 \pi}{\delta}\right)+\ln \operatorname{cth} \frac{\hbar \omega}{2 k_{\mathrm{B}} T}\right\} .
$$

Очевидно, что окончательный результат зависит от выбора постоянной $\delta$. 
При выборе $\delta=2 \pi$ данное выражение можно трактовать как квантово-тепловую энтропию, или кратко QТ-энтропию $S_{\mathrm{QT}}$, ибо она в точности совпадает с эффективной энтропией, полученной в работе [10]. Это обеспечивает согласованность основных результатов предлагаемых нами микро- и макроописаний и их соответствие эксперименту.

$\mathrm{K}$ модификации исходного формального выражения (42) можно подойти и с другой стороны. Объединив в нем оба слагаемых, представим его в виде

$$
-k_{\mathrm{B}} \int d p d q\{\rho(p) \rho(q)\} \ln \{\rho(p) \rho(q)\} .
$$

Нетрудно видеть, что выражение в фигурных скобках - это функция Вигнера для квантового осциллятора в термостате

$$
W(p, q)=\rho(p) \rho(q)=\{2 \pi \Delta q \Delta p\}^{-1} \exp \left\{-\frac{p^{2}}{2(\Delta p)^{2}}-\frac{q^{2}}{2(\Delta q)^{2}}\right\} .
$$

После подстановки в формулу (41) явных выражений (14) и (15) для $\Delta q$ и $\Delta p$ функция Вигнера принимает вид

$$
W(p, q)=\frac{\omega}{2 \pi k_{\mathrm{B}} T_{\mathrm{eff}}} e^{-\varepsilon / k_{\mathrm{B}} T_{\mathrm{eff}}},
$$

где $\varepsilon(p, q)$ - случайная энергия классического осциллятора в QТ-стате с эффективной температурой вида (35).

Замена переменных в фазовом пространстве и переход к безразмерной функции Вигнера с учетом ее нормировки позволяет придать выражению (46) вид QТ-энтропии

$$
S_{\mathrm{QT}}=-k_{\mathrm{B}} \int d \tilde{\varepsilon} \widetilde{W}(\tilde{\varepsilon}) \ln \widetilde{W}(\tilde{\varepsilon}),
$$

где

$$
\widetilde{W}=\frac{\hbar \omega / 2}{k_{\mathrm{B}} T_{\mathrm{eff}}} e^{-\varepsilon / k_{\mathrm{B}} T_{\mathrm{eff}}}
$$

Таким образом, модификация выражения (42) приводит в рамках $\hbar k \mathrm{D}$ к выражениям для QТ-энтропии вида (45) (при $\delta=2 \pi$ ) или (49). С микроскопической точки зрения они обосновывают выражение для эффективной энтропии как макропараметра в равновесной квантовой статистической термодинамике [10]. Заметим, что традиционное выражение для энтропии в КСM оказывается лишь квазиклассическим приближением к предложенной выше QТ-энтропии.

\section{7. НЕКОТОРЫЕ ИТОГИ}

Предыдущее изложение показало, что на основе развиваемой здесь $\hbar k \mathrm{D}$ эффек-

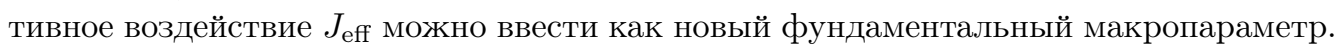
Его преимущество состоит в том, что в данном случае у него есть микроскопический прообраз - оператор стохастического воздействия $\hat{j}$. Более того, через него же в принципе можно выразить основные термодинамические характеристики объектов в тепловом равновесии. Среди них, как известно, наиболее фундаментальными 
являются температура и энтропия. Принято считать, что они не имеют микроскопических прообразов, но при этом обобщенно учитывают стохастическое воздействие окружения на объект. При традиционном изложении о температуре говорят как о "степени нагретости", а об энтропии - как о "мере хаотичности" системы.

При использовании понятия эффективного воздействия этим эвристическим соображениям можно придать наглядный смысл. Для этого обратимся к выражению (35) для $T_{\text {eff. }}$ Из него следует, что эффективное воздействие также является интенсивным макропараметром, характеризующим стохастическое воздействие "теплого" QТ-стата.

C учетом этого нулевому началу равновесной квантовой статистической термодинамики можно придать вид

$$
J_{\text {eff }}=J_{\text {eff }}^{\text {therm }} \pm \Delta J_{\text {eff }}
$$

где $J_{\text {eff }}^{\text {therm }}$ - эффективное воздействие QТ-стата, а $J_{\text {eff и }} \Delta J_{\text {eff }}-$ среднее значение эффективного воздействия объекта и стандартное отклонение от него. Фактически состояние теплового равновесия можно впредь описывать в духе Ньютона, считая, что в таких ситуациях "стохастическое воздействие равно стохастическому противовоздействию".

Обратимся теперь к эффективной энтропии. В отсутствие механического контакта ее дифференциал равен

$$
d S_{\mathrm{eff}}=\frac{\delta Q_{\mathrm{eff}}}{T_{\mathrm{eff}}}=\frac{d U}{T_{\mathrm{eff}}} .
$$

Подставляя в это соотношение выражения для внутренней энергии (32) и эффективной температуры (35), получим

$$
d S_{\mathrm{eff}}=k_{\mathrm{B}} \cdot d\left(\ln \frac{J_{\mathrm{eff}}}{J_{0}}\right)=d S_{\mathrm{QT}} .
$$

Из соотношения (53) следует, что эффективная, или QТ-энтропия, будучи экстенсивным макропараметром, также выражается через $J_{\text {eff. }}$

В итоге оказывается, что две качественно различные характеристики тепловых явлений - эффективная температура и эффективная энтропия - на макроуровне олицетворяют собой наличие в природе двух сторон процесса стохастизации характеристик объекта за счет контакта QT-статом. Они при любых температурах могут быть выражены через один и тот же макропараметр - эффективное воздействие. Этот макропараметр имеет в качестве микроскопического прообраза в $\hbar k \mathrm{D}$ оператор стохастического воздействия, зависящий совместно от постоянных Планка и Больцмана.

Благодарности. Авторы выражают глубокую благодарность редколлегии журнала за предоставленную возможность опубликовать статью в номере, посвященном столетию со дня рождения Н. Н. Боголюбова. Один из нас (А. Д. Суханов) испытывал радость общения с Николаем Николаевичем на протяжении более тридцати пяти лет. Можно утверждать, что исходная концепция Боголюбова о необходимости совместного рассмотрения квантовых и тепловых эффектов, которую он отстаивал с 1939 г., в значительной мере определила направленность данной работы. 
Выражаем также признательность участникам конференции "Renormalization Group and Related Topics" (Дубна, сентябрь 2008 г.) и семинаров ЛТФ им. Н. Н. Боголюбова (ОИЯИ), кафедры теоретической физики РУДН и ИТФ им. Н. Н. Боголюбова НАНУ (Киев) за полезные дискуссии.

Работа выполнена при поддержке РФФИ (грант № 07-06-00239).

\section{Список литературы}

[1] Л. Д. Ландау, Е. М. Лифшиц, Статистическая физика. Ч. 1, Физматлит, М., 2001.

[2] Р. Б. Лафлин, УФН, 170:3 (2000), 292-303; R. B. Laughlin, Rev. Modern Phys., 71:4 (1999), 863-874.

[3] А. Д. Суханов, ЭЧАЯ, 36:6 (2005), 1281-1342.

[4] Д. Н. Зубарев, Неравновесная статистическая термодинамика, Физматлит, М., 1971.

[5] J. Wu, A. Widom, Phys. Rev. E, 57:5 (1998), 5178-5183.

[6] D. T. Son, A. O. Starinets, Ann. Rev. Nucl. Part. Sci., 57:1 (2007), 95-118; arXiv: 0704.0240 .

[7] Н. Н. Боголюбов, Н. М. Крылов, "Об уравнениях Фоккера-Планка", Записки кафедры математической физики АН УССР, 4, 1939, 5-80; Н. Н. Боголюбов, "Об уравнениях Фоккера-Планка", Собрание научных трудов. Т. V, ред. А. Д. Суханов, Наука, М., 2006, 58-137.

[8] Р. Фейнман, Статистическая механика, Мир, М., 1975.

[9] N. N. Bogoliubov, Kinetic equations for the electron-phonon system, E17-11822, JINR, Dubna, 1978; Н. Н. Боголюбов, Собрание научных трудов. Т. V, ред. А. Д. Суханов, Наука, М., 2006, 639-689.

[10] А. Д. Суханов, ТМФ, 154:1 (2008), 183-196.

[11] A. Einstein, Ann. Phys., 9 (1902), 417-433; 11 (1903), 170-187; 14 (1904), 351-362; А. Эйнштейн, "Кинетическая теория теплового равновесия и второго начала термодинамики"; "Теория основ термодинамики"; "К общей молекулярной теории теплоты", Собрание научных трудов. Т. III, Наука, М., 1966, 34-49; 50-66; 67-74.

[12] Дж. В. Гиббс, Основные приниипы статистической механики, Гостехиздат., М., 1946.

[13] А. Д. Суханов, ТМФ, 148:2 (2006), 295-308.

[14] H. Umezawa, Advanced Field Theory: Micro, Macro and Thermal Physics, AIP, New York, 1993.

[15] А. Эйнштейн, "К квантовой теории”, Собрание научных трудов. Т. III, Наука, М., 1966, 328-335.

[16] А. М. Переломов, Обобщенные когерентные состояния и их применения, Наука, М., 1987.

[17] F. Bloch, Z. Phys., 74:5-6 (1932), 295-335.

[18] N. N. Bogoliubov, On Spontaneus Violation of Symmetry in Statistical Mechanics, Physik, Plenarvortr, 37, Weinheim Bergstr, Hamburg, 1973; Н. Н. Боголюбов, Собрание научных трудов. Т. VIII, ред. А. Д. Суханов, Наука, М., 2007, 398-410.

[19] Л. Больцман, "IV. Аналогии с предложениями из области физики, особенно с предложениями теории теплоты", Отрывок из "Лекций о принципах механики", Вариационные принципы механики, Сб. статей классиков науки, ред. Л.С. Полак, Физматгиз, M., 1959, 468-496.

[20] Э. Шредингер, "К принципу неопределенностей Гейзенберга", Избранные труды по квантовой механике, Наука, М., 1976, 210-217.

[21] O. N. Golubjeva, A. D. Sukhanov, Acta Physica Debrecina, XLII (2008), 69-81.

[22] I. I. Hirschman Jr., Amer. J. Math., 79:1 (1957), 152-156.

[23] В. В. Додонов, В. И. Манько, Тр. ФИАН, 183 (1987), 5-70. 